\title{
Segment Specificity of Load Signal Processing Depends on Walking Direction in the Stick Insect Leg Muscle Control System
}

\author{
Turgay Akay, ${ }^{1}$ Björn Ch. Ludwar, ${ }^{1}$ Marie L. Göritz, ${ }^{1}$ Josef Schmitz, ${ }^{2}$ and Ansgar Büschges ${ }^{1}$ \\ ${ }^{1}$ Department of Animal Physiology, Zoological Institute, University of Cologne, 50923 Cologne, Germany, and ${ }^{2}$ Department of Biological Cybernetics, \\ Faculty of Biology, University of Bielefeld, 33501 Bielefeld, Germany
}

\begin{abstract}
In terrestrial locomotion, sensory feedback from load sensors is important for altering ongoing motor output on a step-by-step basis. We investigated the influence of load signals from the leg on motoneuron pools of the thorax-coxa (ThC) joint in the stick insect walking system. Load sensors were stimulated during rhythmic, alternating activity in protractor coxae (ProCx) and retractor coxae (RetCx) motoneuron pools. Alternating activity in the segment of interest was induced by mechanical stimulation of the animal or pharmacological activation of the isolated thoracic ganglia. Load signals from the legs altered the timing of ThC motoneuron activity by resetting and entraining the activity of the central rhythm generating network of the ThC joint. In the front and middle legs, load signals induced or promoted RetCx activity and decreased or terminated ProCx activity. In the hindleg, reverse transitions were elicited, with increasing load terminating RetCx and initiating ProCx activity. Studies in semi-intact walking animals showed that the effect of load on the ThC-joint motoneurons depended on walking direction, with increased load promoting the functional stance phase motoneuron pool (in forward walking, RetCx activity; in backward walking, ProCx activity). Thus, we show that modifications of sensory feedback in a locomotor system are related to walking direction. In a final set of ablation experiments, we show that the load influence is mediated by the three groups of trochanteral campaniform sensilla.
\end{abstract}

Key words: walking direction; campaniform sensilla; sensory feedback; pattern generation; locomotion; reflex reversal

\section{Introduction}

In legged locomotor systems, sensory input signaling leg displacement and force or strain to central neuronal networks is pivotal for generating functional walking motor outputs (Orlovsky et al., 1999; Pearson, 2000; Cattaert and LeRay, 2001; Büschges, 2005). Load information from the legs plays a particularly important role because stable walking requires effective load distribution across all legs during stance (Duysens et al., 2000; Zill et al., 2004). Walking systems must perform multiple tasks (e.g., forward and backward walking, turning, climbing), and which leg muscles will be active during stance changes according to the task. Sensory feedback provided by load sensors must conse-

\footnotetext{
Received Dec. 1, 2006; revised Feb. 14, 2007; accepted Feb. 15, 2007.

T.A. and A.B. were supported by Deutsche Forschungsgemeinschaft Grants Bu 857/6 and 857/8. J.S. was supported by Deutsche Forschungsgemeinschaft Grant Cr58/10-1 and by the Spatial-Temporal Patterns for ActionOriented Perception in Roving Robots project within the European Commission-Information Society Technologies program. We thank Drs. M. Gruhn, S. L. Hooper, T. Mentel, H. Scharstein, and J. Schmidt for helpful discussions in the course of the work. We thank H.P. Bollhagen and S. Marasigan for excellent technical assistance and S. MeyenSouthard and S. L Hooper for style editing.

Correspondence should be addressed to Dr. Ansgar Büschges, Zoological Institute, University of Cologne, Weyertal 119, 50923 Cologne, Germany. E-mail: ansgar.bueschges@uni-koeln.de.

T. Akay's present address: Department of Physiology, Faculty of Medicine, University of Alberta, Edmonton, Alberta, Canada T6G $2 \mathrm{H} 7$.

B. Ch. Ludwar's present address: Department of Neurobiology, Mount Sinai School of Medicine, New York, NY 10029.

M. L. Göritz's present address: Department of Neurobiology and Behavior, Cornell University, Ithaca, NY 14853. D0I:10.1523/JNEUROSC1.5202-06.2007

Copyright $\odot 2007$ Society for Neuroscience $\quad$ 0270-6474/07/273285-10\$15.00/0
}

quently be differentially processed depending on the walking behavior being performed. This may lead to the hypothesis that task-dependent alterations in the processing of load signals is relevant for the generation of different walking motor outputs, e.g., forward versus backward walking. This point of view is supported by the fact that modifications in processing of sensory feedback signals occur in relation to the generation of walking. Movement signals from limbs activate resistance reflexes in standing and contribute to the control of active movements by reinforcing the actual motor output during walking. This represents a state-dependent reversal in processing of sensory feedback signals (reflex reversal) (DiCaprio and Clarac, 1981; Bässler, 1986, 1988; Pearson and Collins, 1993). Phase dependency of processing of sensory signals has also been described regarding stance and swing phases of the walking motor output (Angel et al., 1996). Potential task-dependent modifications are as yet unknown.

In stick insect legs, the main load receptors are the campaniform sensilla (CS), knob-like sense organs located in the cuticle that are activated by cuticular strain (for review, see Zill et al., 2004) induced both by passive loading of the leg and during muscle driven movement (Pringle, 1938; Hofmann and Bässler, 1982, 1986; Delcomyn, 1991). Input from the CS fields of the trochantero-femur substantially alter leg motor output (Bässler, 1977; Schmitz, 1993; Schmitz and Stein, 2000; Akay et al., 2001, 2004) and help coordinate the motoneuron activities of the indi- 
vidual central pattern generating networks that control the different joints. The femoral CS afferents alter, in particular, the central rhythm generator (CRG) of the femur-tibia (FT) joint so as to promote and enhance flexor tibiae motoneuron activity during stance (Akay et al., 2001). Signals from the trochanteral CS (trCS), alternatively, change thorax-coxa (ThC) joint CRG activity (in forward walking) by promoting retractor coxae motoneuron activity during stance (Akay et al., 2004). The neural mechanisms responsible for these changes are as yet unknown.

We show here that (1) leg load signals alter the activity of the central rhythm generating networks of the ThC joints of all legs, (2) this influence is segment specific, (3) the effect of the load signal depends on leg walking direction, and (4) these signals derive primarily from the trCS. This work demonstrates that the effect of sensory input on central pattern generating network activity varies as a function of walking state (forward vs backward walking).

\section{Materials and Methods}

The experiments were performed on adult female stick insects (Carausius morosus) from breeding colonies at the University of Cologne and Bielefeld University. All experiments were performed under daylight conditions and at room temperature $\left(20-22^{\circ}\right)$. Two preparations were used. In the first, all legs were removed except one, and the animal's responses to sensory input were investigated in three activity states: (1) when the insects were quiescent ("inactive" state), (2) when they were activated by touching the abdomen or head with a paint brush ("active" state), and (3) when the central rhythm generating networks had been activated by adding the muscarinic agonist pilocarpine to the isolated thoracic ganglia ("rhythmic preparation") (for additional details, see Büschges et al., 1995). The second preparation simulated a semi-intact walking condition in which five legs were allowed to walk freely on a slippery glass plate (Graham and Epstein, 1985) while either one middle or one hindleg was held in a prolonged stance phase ("walking" state).

Preparation. The single-leg preparations used to test the trCS influence on ThC-joint motoneurons were prepared as follows. Animals with only one front, middle, or hindleg left attached were glued dorsal side up to a foam platform with dental cement (Protemp II; 3M ESPE, St. Paul, MN). The leg was then fixed on the platform perpendicular to the body axis and extended over the rim of the foam platform. The coxa was embedded in dental cement so that the ThC joint could not move. Care was taken not to cover the CS located on the trochanter and the proximal femur with cement. A small window was cut distal from the CS into the femoral cuticle, and all femoral tissue was severed to exclude sensory influences from the more distal part of the leg. A cut along the midline of the mesothoracic and metathoracic tergum was made, and the two sides were folded apart and fixed with insect pins. Gut and connective tissue were removed to expose the ventral nerve cord, taking care to leave as much of the tracheation as possible intact, and the inside of the insect was filled with saline (Weidler and Diecke, 1969). Leg nerves were identified according to Marquardt (1940) and Graham (1985). All lateral nerves were cut except for nervus cruris. The activity of the motoneurons that innervate the protractor (ProCx) and retractor ( RetCx) coxae muscles was monitored by extracellular recordings using hook electrodes (modified after Schmitz et al., 1988) on the lateral nerve stumps nl2 and nl5 (ProCx and RetCx, respectively). The CS were stimulated by caudal bending $(200-340 \mu \mathrm{m})$ of the femur horizontally using a piezoelectric element or pen motor (according to Schmitz, 1993). In these preparations, the effect of CS stimulation on ThC-joint motoneurons was first tested in resting, quiescent animals (inactive state). The animals were then activated by touching their abdomen with a paintbrush. In this state (active state), the animals perform random leg movements or walk if the legs are not restrained. In animals in which the legs are restrained or denervated, the motoneurons innervating antagonistic leg muscles show alternating activity (for review, see Bässler and Büschges, 1998). The CRG networks in the thoracic nervous system were also pharmacologically activated by applying the muscarinic agonist pilocarpine (Büschges et al., 1995). In this case (rhythmic preparation), all nerves and connectives attached to thoracic ganglion of interest (prothorax, mesothorax, or metathorax) were cut with only the innervation of the CS left intact.

In the walking experiments, the insects walked on a grease-coated surface with reduced friction (Epstein and Graham, 1983; Graham and Epstein, 1985; Gruhn et al., 2006). The insect was fixed on a 4-mm-wide balsa wood rod suspended above the slippery surface at a height (5-8 $\mathrm{mm}$ ) at which the femurs had a natural horizontal position when FT joint angle was at $90^{\circ}$. Indentations carved on the dorsal side of the rod at each leg position allowed free coxa movements during walking. For load sensor stimulation on middle and hindlegs, the leg was fixed to the rod with dental cement at its coxal base at positions of 90 and $110^{\circ}$, respectively, similar to the procedure described for the middle leg by Schmitz (1993). The femur was cut off close to the FT joint. The animal's thorax was opened from the dorsal side according to Schmitz (1993). All ipsilateral nerves of the segment under investigation except for the nervus cruris, $\mathrm{nl} 5$, and $\mathrm{nl} 2$ were cut or crushed close to the ganglion. The trochanterofemoral CS were stimulated, and motor activity in lateral nerves nl5 and $\mathrm{nl} 2$ were recorded as in the one-legged experiments described above. CS stimulation was confirmed in the resting animal by local reflex activation of coxal motoneurons during femur bending (cf. Schmitz, 1993). Walking movements were induced by tactile stimulation of the animal's abdomen or head, depending on desired walking direction (Graham and Epstein, 1985). An animal was considered to walk forward or backward when the five unrestrained legs exhibited a clear forward or backward stepping pattern on the slippery surface. In addition, the motor activity of two muscles that have very different coordination patterns in forward and backward walking (the retractor coxae and levator trochanteris muscles) (Graham and Bässler, 1981; Graham and Epstein, 1985) were also electromygraphically monitored in one adjacent ipsilateral leg (e.g., when load sensors on the right hindleg were stimulated, the right middle leg was monitored).

Data analysis. Extracellular recordings and the stimulation traces were stored on a digital audiotape recorder [PC 116 (Sony, Toyko, Japan) or DTR 1800 (Bio-Logic, Claix, France)]. In parallel, analog-to-digital conversion was performed (sampling rate, $12.5 \mathrm{kHz}$ ) with CED 1401plus interface (Cambridge Electronic Design, Cambridge, UK). The recordings were analyzed with Spike2 software (versions 3.13-4.03; Cambridge Electronic Design). Statistical evaluation of the data and plotting of the graphs were performed with Excel 97 (Microsoft, Seattle, WA). In text, $N$ is the number of experiments, and $n$ is sample size.

\section{Results}

In resting stick insects (C. morosus) leg sensory signals reporting increased caudal load terminate ongoing tonic ProCx activities and activate RetCx (Schmitz, 1993; Schmitz and Stein, 2000). Bending the femur in a rostral direction has the opposite effect, activating ProCx and inhibiting RetCx motoneurons. This influence is mediated by the CS located on the anterior and the posterior side of the femur and trochanter (Hofmann and Bässler, 1982). For the middle leg, Schmitz (1993) showed that this influence persists when animals walk forward on a treadmill. We expanded this study by including the other thoracic segments and examining both forward and backward walking animals.

\section{Stimulation of middle leg CS during rhythmic mesothoracic coxal motoneuron activity}

The effect of middle leg CS stimulation on rhythmic mesothoracic coxal motoneurons was investigated in (1) the active state of the stick insect locomotor system induced by tactile stimulation of the animal with a small brush (Bässler, 1993) and (2) animals in which rhythmic leg motoneuron activity was pharmacologically induced by applying $1 \times 10^{-4}$ to $5 \times 10^{-4} \mathrm{M}$ pilocarpine to the ganglion (Büschges et al., 1995). When ThC motoneuron rhythmicity was induced by tactile stimulation, caudal strain on the femur most often induced cessation of ProCx activity and 


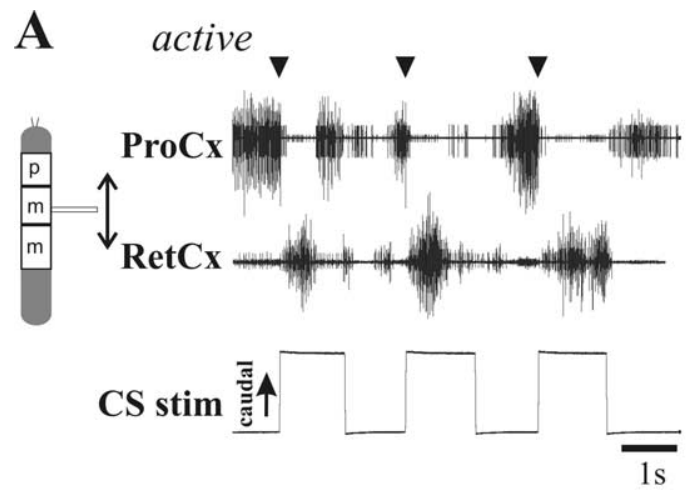

B

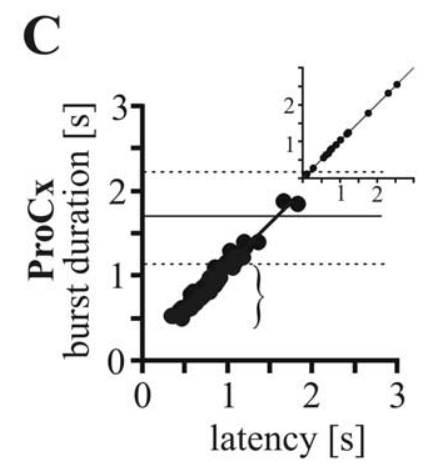

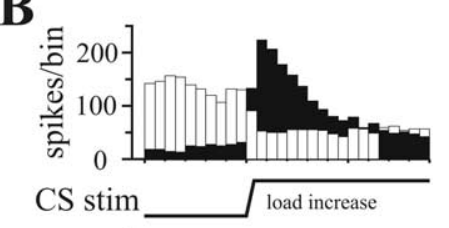

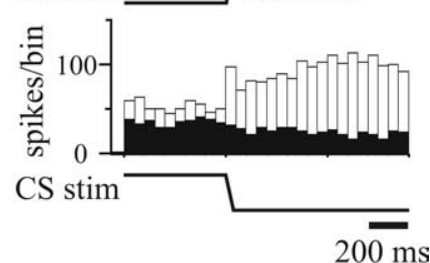

D

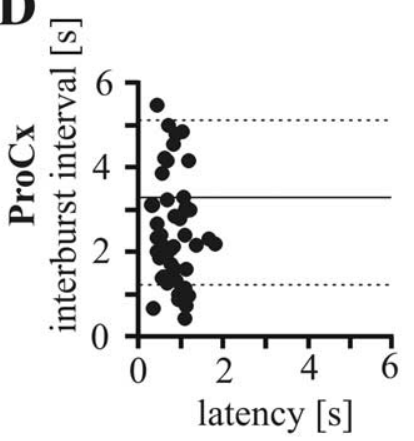

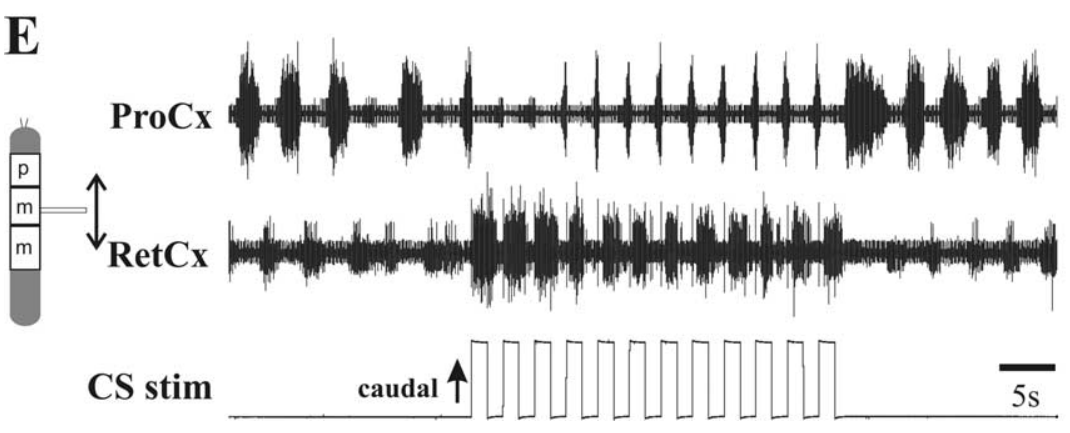

Figure 1. Effect of $\mathrm{CS}$ stimulation on the ThC-joint motoneuron activity in insects with activated locomotor systems. A, Extracellular recordings from ProCx (top trace; nerve $\mathrm{nl} 2$ ) and RetCx (middle trace; nerve $\mathrm{n} 15$ ) nerves during $\mathrm{CS}$ stimulation (bottom trace) of a middle leg in a stick insect activated by tactile stimulation. CS stimulation (upward deflection indicates caudal bending of the femur) induced a transition from ProCx to RetCx activity (arrowheads). $\boldsymbol{B}$, PST histograms (bin width, $50 \mathrm{~ms}$ ) illustrating that ProCx activity (white bars) decreased and RetCx activity (black bars) increased during $\mathrm{CS}$ stimulation by caudal bending of the femur (top histogram; $n=27$ ) in one tactilely activated stick insect. Rostral bending on average increased ProCx activity but only slightly decreased RetCx activity (bottom histogram; $n=27$ ) C, Plot of the duration of ProCx bursts in which a stimulus was applied as a function of latency between ProC $x$ burst onset and the stimulation onset during pilocarpine-induced rhythmic activity in four animals $\left(n=46 ; R^{2}=0.96\right)$ and in one tactilely activated animal (inset, $n=17 ; R^{2}=0.99$ ). Note that, when stimuli occur early in the ProCx burst, the bursts (bracket) were significantly shorter than under control conditions. Horizontal lines indicate mean (solid line) \pm SDs (dashed lines) of ProCx burst durations without stimuli $(N=4 ; n=140)$. D. Plot of the interburst intervals after the terminated Pro $\mathrm{C}$ bursts (in $C$ ) as a function of latency between Pro $\mathrm{C}$ burst onset and stimulation onset during pilocarpineinduced rhythmic activity in four animals $(n=46)$. Note that termination of the Pro $\mathrm{Cx}$ bursts by the $\mathrm{CS}$ stimuli did not alter the following interburst interval, indicated by no correlation $\left(R^{2}=0.01\right)$ and comparable interburst intervals after $C S$ stimulation versus the control intervals. $\boldsymbol{E}$, Entrainment of ThC-joint motoneuron activity by load signals from the leg. Sample recording of Pro $\mathrm{Cx}$ (top trace) and RetCx (middle trace) activity in the middle leg during repetitive stimulation with a higher frequency than the inherent frequency of the centrally generated rhythm in the presence of pilocarpine (for details, see Results).

switching activity from ProCx to RetCx $(N=9 ; n=155)$. In contrast, RetCx burst duration was not affected by load signals from the femur (data not shown). A closer look at Figure $1 A$ reveals the absence of the CS influence on the RetCx burst duration, given their ongoing activity during load stimulation (cf. Delcomyn, 1991). That is, during the first two stimulation trials in Figure $1 A$, the RetCx bursts terminate before the femur is released from the caudal bending. Releasing the femur from the caudal bending did not have a systematic influence (Fig. $1 A, B$ ) except for increasing ProCx motoneuron activity.

In the cases when ProCx activity was terminated by a caudal-bending stimulus, ProCx burst duration depended linearly on the latency of the stimulus after burst beginning. This is exemplified in Figure $1 C$ by a plot of ProCx burst duration versus CS stimulus latency relative to time of ProCx burst begin (main plot, pilocarpine-induced activity, $N=4, n=$ 46 ; inset, data from an animal activated by tactile stimulation). The earlier the CS stimulus was given after the burst begins (bracket), the shorter was the burst duration compared with the control (without stimulation) experiments (horizontal solid line, mean; horizontal dashed lines, \pm SDs). The fact that the stimulated ProCx burst durations are shorter than control ProCx bursts and that there is a linear correlation between ProCx burst duration and stimulus latency show that ProCx bursts are indeed terminated by CS stimulation.

This CS-induced switch of motoneuron activity from ProCx to RetCx could be mediated by input acting either directly onto the ThC-joint motoneurons or indirectly by the input altering the activity of the entire ThC-joint CRG. The latter case would predict that CS stimulation could reset or entrain ThC-joint motoneuron rhythmic activity. To test this possibility, we plotted the subsequent interburst interval versus the latency of the trCS stimulus from ProCx burst onset for the ProCx bursts that were terminated by CS stimulation. If the CS input was simply terminating the ProCx motoneuron burst but not resetting the ThC-joint CRG because the input was applied at varying times to

initiated RetCx activity (Fig. $1 A$ ). Loading the femur by caudal bending terminated ProCx motoneuron activity and induced RetCx motoneuron activity in $82 \%$ of the trials [Fig. $1 \mathrm{~B}$, peristimulus time (PST) histograms of ProCx and RetCx motoneuron activity] $(N=9 ; n=158)$. CS stimulation had the same effect on ThC-joint motoneuron activity in the pharmacologically activated isolated (except for the CS innervation) mesothoracic ganglion in $82 \%$ of the cases either terminating ProCx activity or the CRG cycle period, one would expect the subsequent interburst interval to depend on stimulus latency (if the input occurred early in the ProCx burst phase, one would expect a long latency to the next burst, but if it occurred late in the ProCx burst phase, one would expect a short latency). Alternatively, if the CRG was being reset by each CS stimulation, the time to the next ProCx burst (the interburst interval to the subsequent burst) should be the same regardless of when the stimulus was applied. 
In all experiments (Fig. 1D), interburst intervals were similar to control values (horizontal solid line, mean; horizontal dashed lines, \pm SDs) and were not correlated with stimulus latency. This constancy of interburst interval regardless of where in the burst cycle the ProCx burst was terminated indicates that, in each case, the ThC-joint CRG was being reset, clearly showing that signals from load sensors affect the CRG of the ThC joint. This access of load signals to the ThC-joint central rhythm generator was further shown by load signals being able to entrain ongoing pilocarpine-induced rhythmic activity in mesothoracic ProCx and RetCx motoneurons (Fig. 1E). Thus, in the mesothoracic segment, signals from leg load sensors have access to the ThCjoint CRG and promote transitions in activity from ProCx to RetCx motoneurons.

\section{Stimulation of front leg CS during rhythmic prothoracic} coxal motoneuron activity

Load increases or decreases applied to the front leg during alternating prothoracic coxal motoneuron activity $(N=9)$ (activated either tactilely or pharmacologically) had the same influence on prothoracic activity as did middle leg stimulation on mesothoracic activity (Fig. $2 A, B$ ). In brief, in tactilely activated animals, an increase in load by caudal bending of the femur induced in $54.8 \%$ of the cases either termination in ProCx activity or a transition from ProCx to RetCx activity $(n=148)$. In $84.8 \%$ of the rhythmic preparations, either a termination in ProCx activity or a transition from ProCx to RetCx activity was induced $(n=171)$. Thus, as in the mesothoracic segment, CS stimulation terminated ProCx activity and started RetCx activity. Again as in the mesothoracic segment, this change in ProCx activity resulted in ProCx burst duration being correlated with the delay from burst beginning to stimulus onset (Fig. 2C, data from one tactilely activated animal). Prothoracic segment load sensory signals thus have the same influence on prothoracic ThC-joint motoneuron activity as mesothoracic load signals have on mesothoracic activity.

\section{Stimulation of hindleg CS during rhythmic metathoracic coxal motoneuron activity}

As in the mesothoracic and prothoracic segments, in the inactive state, caudally directed strain induced an increase of metathoracic RetCx activity. However, in active animals, hindleg load signals exerted a reversed influence on metathoracic ThC-joint motoneurons, most often either terminating RetCx activity alone (Fig. 3Ai, first stimulus) or inducing a transition from RetCx to ProCx activity (Fig. 3Ai, second stimulus). The same was true for metathoracic preparations activated by pilocarpine (Fig. 3Aii). Caudal bending of the femur during RetCx activity elicited a transition from RetCx to ProCx activity in 43\% of mechanically activated animals and $82 \%$ of pilocarpine-activated metathoracic ganglions. When this stimulus was presented during ongoing ProCx motoneuron activity, in only $10-20 \%$ of the cases was a termination of ProCx motoneuron activity and a subsequent transition to RetCx activity observed. Releasing the femur from caudal bending had no systematic effect on metathoracic ThCjoint motoneurons. Peristimulus time histograms of ProCx and RetCx motoneuron activity (Fig. 3B) confirm that caudally directed femoral bending usually increased ProCx motoneuron activity and decreased RetCx activity.

Figure $3 C$ shows that RetCx burst durations significantly correlated with the latency of the CS stimulus relative to RetCx burst beginning $(N=3, n=39$; main plot shows all data, and inset is data from one animal activated by tactile stimulation). RetCx burst duration was shorter than control RetCx burst durations

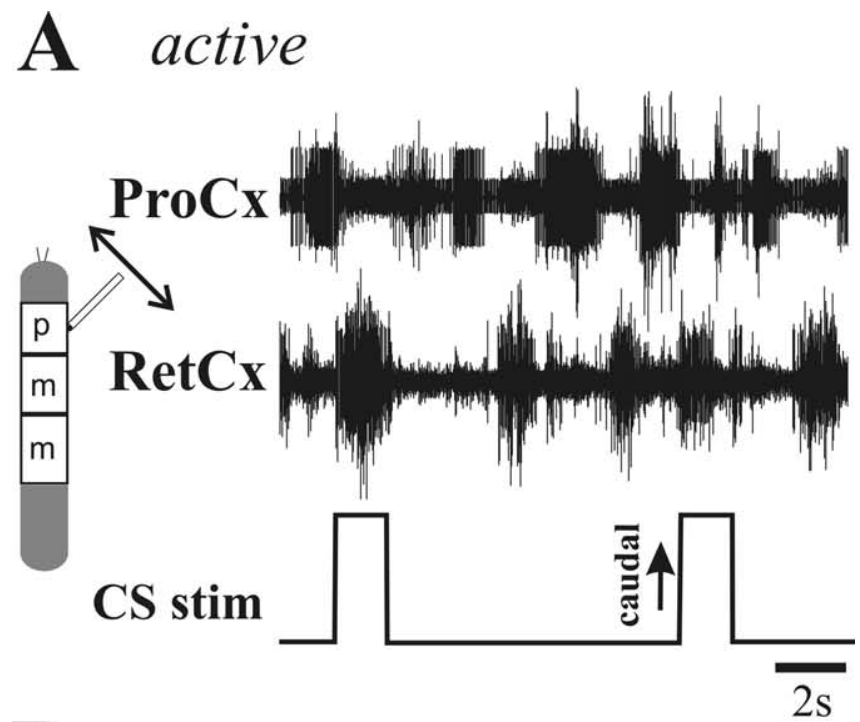

B

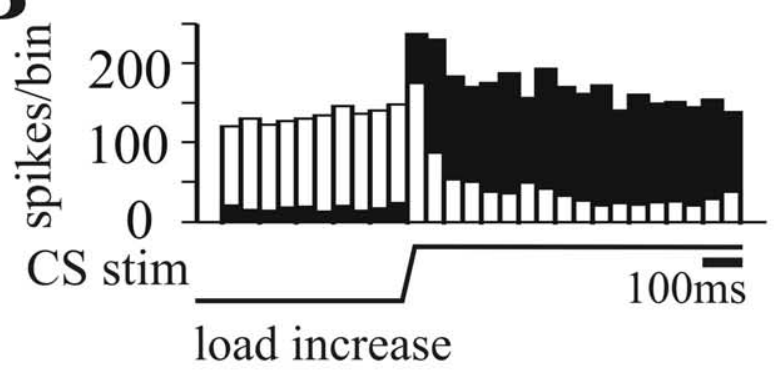

C

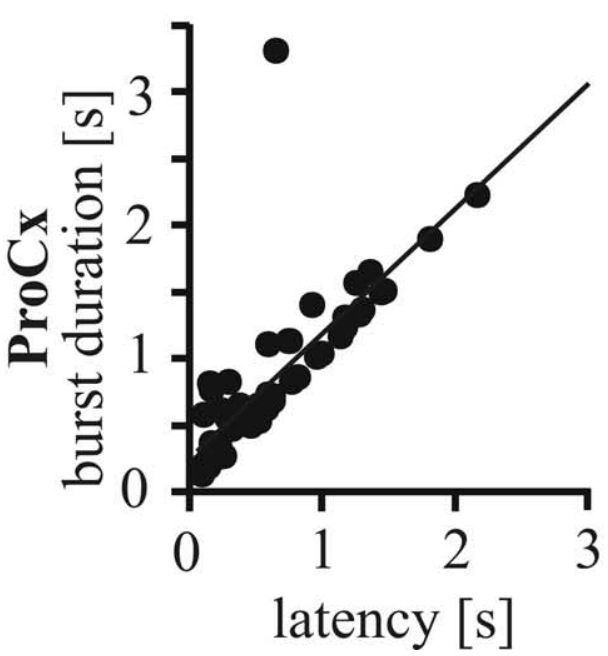

Figure 2. A, Extracellular recordings from ProCx (top trace; nerve $\mathrm{nl} 2$ ) and RetCx (middle trace; nerve $\mathrm{nl5}$ ) nerves during $\mathrm{CS}$ stimulation (bottom trace) of a front leg in a stick insect activated by tactile stimulation. CS stimulation (upward deflection indicates caudal bending of the femur) induces transition from Pro $C x$ to RetCx activity. $B$, PST histograms (bin width, 50 ms) illustrating that ProCx activity (white bars) decreased and RetCx activity (black bars) increased during $C S$ stimulation by caudal bending of the femur $(n=52)$ in one tactilely activated stick insect. $C$, Plot illustrating that the ProCx burst durations were significantly correlated with the latency of the onset of CS stimuli in fore legs $\left(N=3 ; n=55 ; R^{2}=0.71\right)$ in tactilely activated animals.

(horizontal solid line, mean; horizontal dashed lines, \pm SDs) when the CS stimuli occurred early in the burst cycle (bracket) and the RetCx burst durations linearly correlated with stimulus onset latency, indicating that CS stimuli terminated the RetCx 

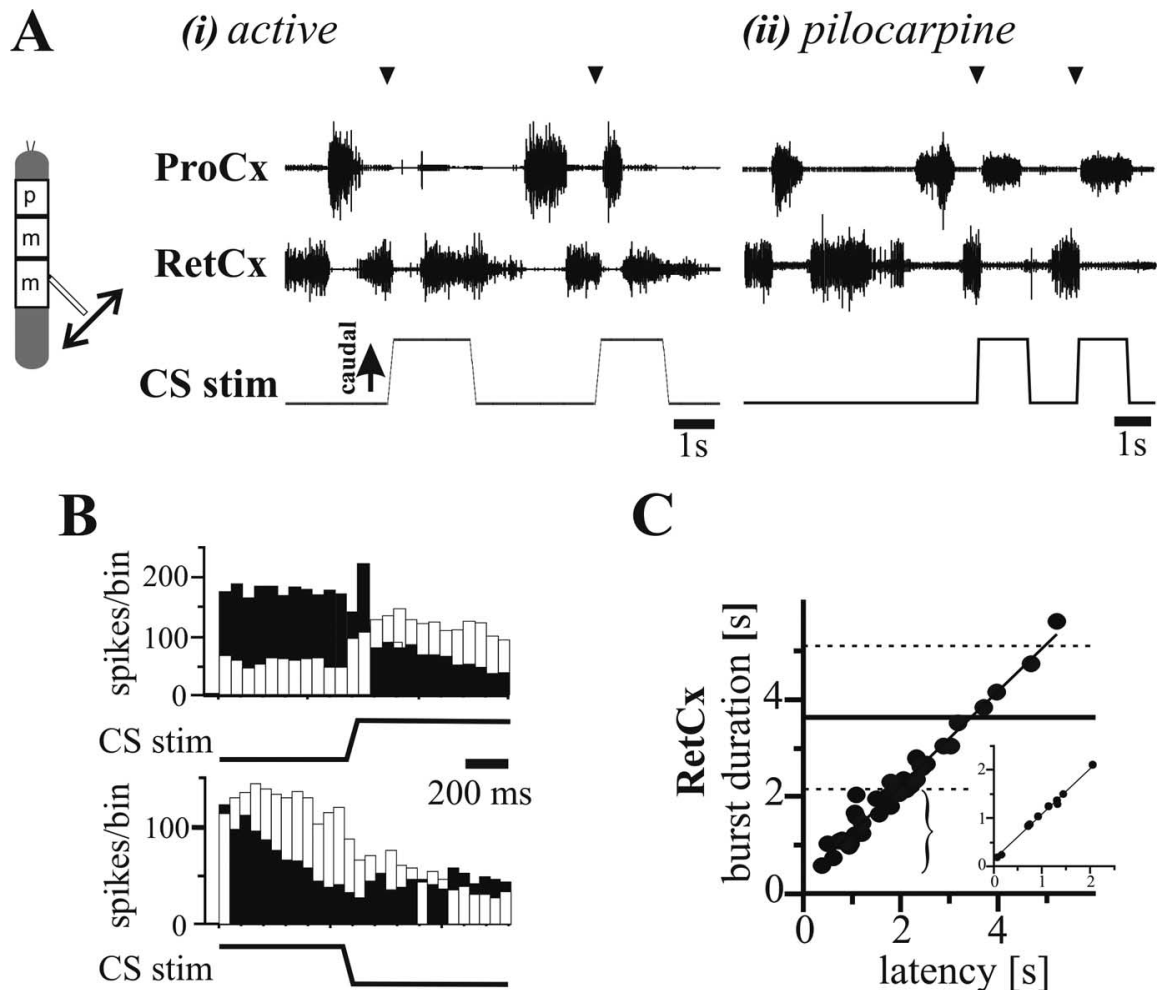

C

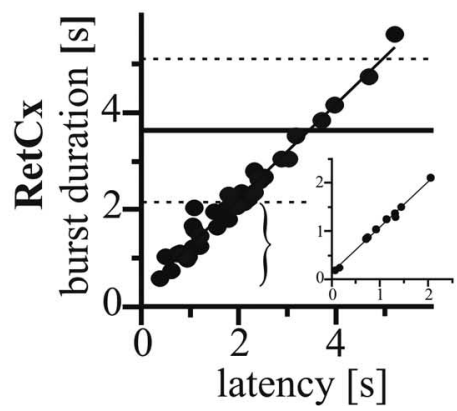

Figure 3. Reversed effect of load signals on ThC-joint motoneuron activity in the metathoracic segment. $\boldsymbol{A}$, Extracellular recordings from ProCx (top traces) and RetCx (middle traces) nerves during stimulation of load sensors of the hindleg (bottom traces) in experimental animals activated by either tactile stimulation (i) or $5 \times 10^{-4} \mathrm{~m}$ pilocarpine (ii). Stimulation of load sensors induced a transition from RetCx to ProCx activity in both conditions (arrowheads). B, PST histograms (bin width, 50 ms) illustrating that, in the metathoracic segment, ProCx activity (white bars) increased and RetCx activity (black bars) decreased during CS stimulation by caudal bending of the femur (top histogram; $n=16$ ) in one tactilely activated stick insect. On average, rostral bending of the femur does not show stimulation-related changes in ThC-joint motoneuron activity (bottom histogram; $n=$ 16). C, Plot of RetCx burst duration in which the stimulus was applied versus the latency between burst onset and stimulation onset $\left(N=3\right.$ animals; $\left.n=39 ; R^{2}=0.96\right)$ in the pilocarpine-induced rhythmic preparation and in the tactilely activated animal (inset; $n=9 ; R^{2}=0.99$ ). Note that, when stimuli occur early in a RetCx burst, the burst was significantly shorter (bracket) than the control bursts. Horizontal lines indicate mean (solid line) \pm SDs (dashed lines) of RetCx burst durations without stimuli $(N=3$; $n=39)$.

bursts. trCS stimulation was also able to reset and entrain pilocarpine-induced metathoracic segment rhythmic activity (data not shown).

In summary, the data shown so far indicate that, when alternating activity was generated in coxal motoneurons, load signals from the leg influence ThC-joint motoneuron activity in the same way in front and middle legs, regardless of whether the animal is at rest or walking (Schmitz, 1993): increasing load initiated RetCx activity and terminated ongoing ProCx activity. During ongoing rhythmic activities in hindlegs, however, the influence of load signals is reversed compared with both the effect in the resting animal and the effect in the prothoracic and mesothoracic segments. With increasing load, ProCx activity is initiated.

CS influence depends on walking direction, not segment (pro, meso, meta) identity

Bässler et al. (1985) reported that, when the other legs had been removed, the preferred walking direction of single legs varied in a segment-specific manner, with single front or middle legs preferentially producing forward stepping and hindlegs backward stepping. In the experiments presented here, all legs except for the one under consideration had similarly been removed. It is therefore possible that the reversal of the load influence on ThC-joint mo- toneurons in the prothoracic and mesothoracic segments compared with the metathoracic segment shown above may depend on the preferred walking direction of the different segments rather than on segment identity per se. To test this hypothesis, we stimulated load sensors on hindlegs $(N=7)$ and middle legs $(N=3)$ during forward and backward walking in intact animals. The animals walked with five legs on a slippery glass plate either forward or backward (for how walking direction was defined, see Materials and Methods) while the leg of interest was kept in a prolonged stance phase.

Figure $4 A$ shows two typical ProCx and RetCx nerve recordings from a hindleg of a forward $(A i)$ and backward (Aii) walking insect. Notice that, when load sensors are stimulated, the induced change in coxal motoneuron activity reverses depending on the walking direction of the other five legs: during forward walking, load signals cause a switch from ProCx to RetCx motoneuron activity, whereas during backward walking the same input causes a switch from RetCx to ProCx activity. Figure $4 B$ shows PST histograms from a single experiment of ProCx (white bars) and RetCx (black bars) motoneuron activity for 18 stimuli during forward walking and 33 stimuli during backward walking. The influence of CS stimulus on motoneuron activity clearly reverses as a function of walking direction.

As in the reduced preparation described above, rhythmic CS stimulation was able to entrain the walking CRG, and the effect of this entrainment again depended on walking direction (Fig. 5). Figure 5 shows the effect of rhythmic CS stimulation during backward walking; the walking pattern becomes entrained to the stimulus with the ProCx motoneurons firing during the caudal portion of the CS stimulation. When the same CS stimulation is performed during forward walking, however, the walking pattern becomes entrained to the stimulus with the RetCx motoneurons firing during the caudal portion of the CS stimulation.

These results support the idea that the influence of load signals on ThC-joint motoneuron activity in the hindleg depends on walking direction. To validate this hypothesis further, we performed similar experiments with the middle leg. During forward walking (Fig. 6Ai), stimulation of middle leg load sensors caused a transition from ProCx to RetCx similar to that seen in mechanically or pharmacologically activated single-legged animals (Figs. 1,2). However, when the insect walked backward (Fig. 6 Aii), the load influence reversed, causing a transition from RetCx to ProCx. The frequencies of transitions in motor activity during changes in load are summarized in Figure $6 B$ for seven hindlegs and three middle legs. When load was either increased $(B i)$ or decreased (Bii), the effect reversed (that is, switched from terminating RetCx and starting ProCx to terminating ProCx and starting RetCx or vice versa) when walking direction reversed. One important difference between the single-legged and intact ani- 
mals, for both hindlegs and middle legs, was that, in the intact animals, release from caudal loading had a stronger effect on coxal motoneuron activity. During forward walking, 58 and $92 \%$ of hindleg and middle leg releases, respectively, resulted in changes in motoneuron activity in the intact case (Fig. 6 Bii, left), whereas in the single-legged preparations, the values did not exceed $50 \%$. A similar high incidence of strain release altering motoneuron activity was also present during backward walking (Fig. 6 Bii, right).

It is important to note again that the dependence on walking direction shown in Figures 4-6 is consistent with previous work (Bässler et al., 1985) and the data reported above from activated, reduced preparations. In single-leg preparations, isolated front or middle legs generate forward walking patterns and isolated hindlegs backward walking patterns. As a result of this opposite walking direction, locomotor networks in the metathoracic ganglia show a reversed response to leg load signals compared with the responses of the networks in prothoracic and mesothoracic ganglia.

\section{The reversal of CS influence occurs immediately when walking direction changes}

The above experiments show that the effect of CS stimulation reverses when walking direction reverses, but, because these data were taken over prolonged periods of forward or backward walking, they do not show the time course of the reverse effect when walking direction changes. To investigate this issue, we applied periodic CS stimulation and induced a change in walking direction by first inducing forward walking by tactile stimulation of the abdomen and then backward walking by tactile stimulation of the antennae (Fig. 7). The influence of the load signals changed immediately from CS input terminated ProCx activity and stimulated RetCx activity (forward walking) to stimulating ProCx activity and terminating RetCx activity (backward walking) when walking direction changed.

\section{Ablation experiments}

To determine which of the trochantero-femoral load sensors were responsible for the transitions in bursting activities, we successively ablated the campaniform sensilla fields on the trochanter and femur, the trCS and fCS, respectively, in the single-legged preparations both at rest and during alternating coxal motoneurons activity (Bässler, 1977; Akay et al., 2001, 2004). Removal of the fCS $(N=3)$ did not affect reflex activation of coxal motoneurons at rest (data not shown) or alter the initiation incidence of the most often detected changes in motor activity for a given CS stimuli (Fig. $8 \mathrm{~A}$ ). In contrast, removal of both fCS and the trCS $(N=3)$ almost completely abolished the effects of CS stimulation (Fig. $8 \mathrm{~B}$ ). Removing the trCS alone in three other preparations also significantly decreased response frequency to CS stimulation
(Fig. $8 C$ ). These ablation experiments thus indicate that strain information from the trCS is primarily responsible for the changes in ThC-joint motoneuron activity in response to CS activation shown here.

\section{Discussion}

We have shown here that leg load sensors play a crucial role in determining thoraco-coxal ProCx and RetCx motoneuron activity in rhythmically active animals. Ablation experiments showed that this input primarily arises from the campaniform sensilla fields on the trochanter. In front and middle legs, increased caudal loading generally terminated ProCx motoneuron activity and initiated RetCx motoneuron activity. Hindleg stimulation had the opposite effect. Common to all segments was that load signals could reset and entrain rhythmic activity in ThC-joint motoneurons, indicating that load signals have access to the ThC-joint CRG. Experiments in which one leg was stimulated while the remaining five legs walked unrestrained showed that the effect of leg loading depended on the walking direction, not segment identity, always assisting functional stance muscle activity (cf. Schmitz and Haßfeld, 1989). During forward walking, stimulation of load sensors induced a transition from ProCx to RetCx 


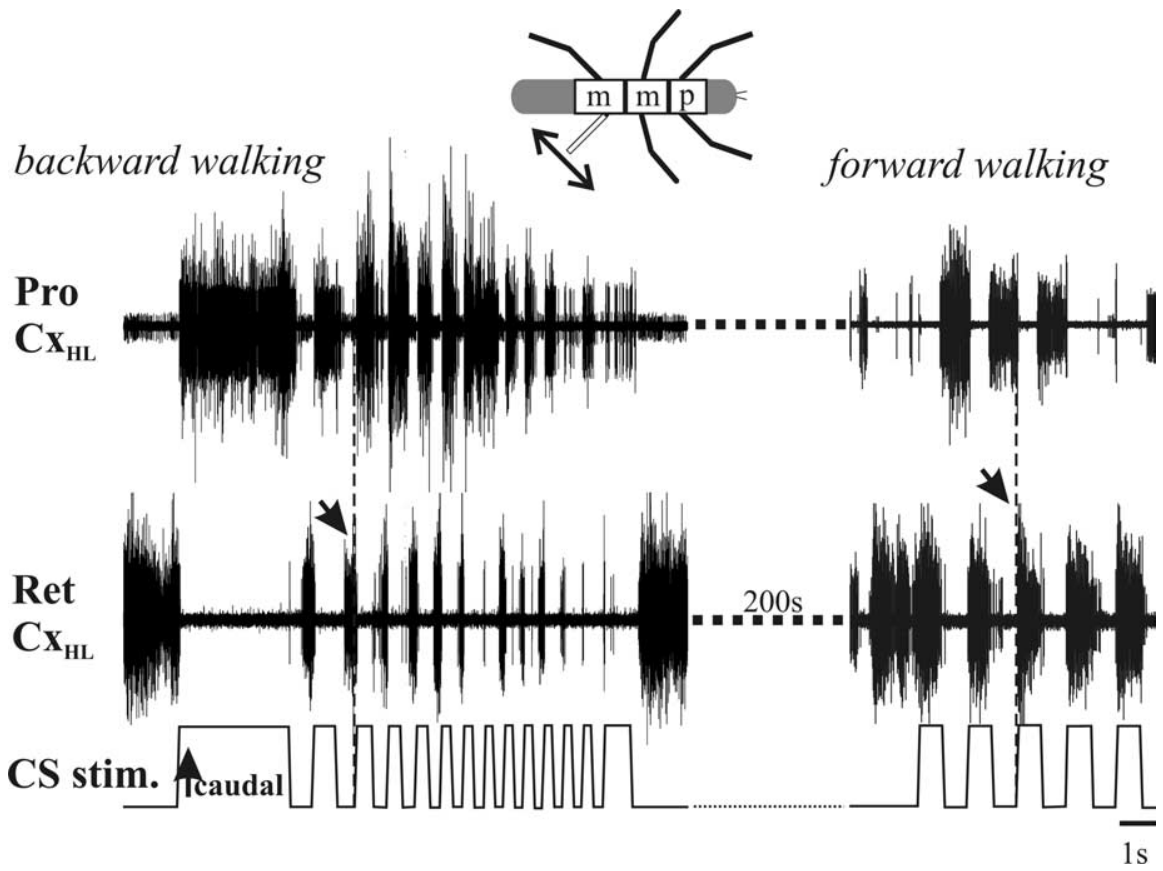

Figure 5. Phase of entrainment of rhythmic ThC-joint motoneuron activity by rhythmic stimulation of hindleg load sensors depends on walking direction. During backward walking (left), entrainment results in ProCx activity being in phase with caudal bending of the femur. In contrast, during forward walking (right), entrainment results in RetCx activity being in phase with caudal bending of the femur. Arrows at stippled vertical lines each denote one transition in activity of coxal motoneurons induced by CS stimulation in backward and forward walking.

activity, whereas during backward walking, a transition from RetCx to ProCx occurred. Thus, in all legs, increased load induced a transition from swing to stance.

\section{Influence of load information on the patterning of ThC-joint motoneuronal activity}

Leg load signals play an important role in controlling step timing and amplitude in walking animals in both vertebrates and invertebrates (Duysens et al., 2000; Zill et al., 2004). In particular, leg load signals reinforce or prolong stance phase motor activity (Bässler, 1977; Cruse, 1985; Whelan et al., 1995; Hiebert et al., 1996; Akay et al., 2001). In a previous study, we showed that trCS sensory signals mimicking load increases initiated and increased retractor activity in an isolated "walking middle leg preparation" (Akay et al., 2004). However, it remained unclear how coxal motoneuron activity was affected. We have shown here that load signals affect timing in coxal motoneuron activity by altering the operation of the entire ThC-joint CRG network. This possibility had been suggested previously as one plausible mechanism for the effects of leg loading and was implemented and successfully tested in a neuromechanical simulation of stick insect walking (Ekeberg et al., 2004).

At this point, our data indicate that primarily trCS supply the load signals underlying the observed influence on ThC motoneuron activity. However, in the ablation experiments, some contribution of the fCS signals to ThC-joint motoneuron activity also became apparent. When only the trCS were ablated (Fig. 8C), an influence of the femur bending on the ThC-joint activity was still apparent but at a significantly reduced level. Given that the ablation of the trCS was complete indicates that fCS signals can influence ThC-joint motoneuron activity but only when trCS signals are not present. Ablation of the fCS alone would thus not result in a measurable effect as in Figure $8 \mathrm{~A}$, but, when the trCS are ab- lated, the fCS could still provide some influence as shown in Figure 8C. This corroborates previous results (Akay et al., 2001) showing that fCS signals influence FT-joint motoneuronal activity (reinforcing flexor activity by altering FT-joint CRG network activity), in which trCS stimulation had no detectable influence. It thus appears that, despite the close colocalization of the trCS and the fCS afferents at the trochantero-femoral rim, a functional segregation exists between the two, with fCS altering FT-joint motoneuron activity and trCS altering ThC-joint motoneuron activity. Leg load signals thus affect stick insect walking motor output by acting on two leg joint rhythm generators, the FT and the ThC CRG. These are also the joints that produce the main torques responsible for the forward movement of the body (Bartling and Schmitz, 2000).

\section{Reversal of the CS effect in the hindleg}

Our findings shed light on the differences between the serially homologous segmental ganglia controlling the legs of a walking insect. When alternating activity was generated in RetCx and ProCx motoneurons in isolated front and middle legs, load signals activated RetCx motoneurons and terminated ProCx motoneuron activity, whereas the reverse was true in the hindleg. However, this difference was not attributable to segment identity but instead to the preferred walking direction of the legs of the different segments when isolated: in reduced, activated preparations, the metathoracic ganglion is in a "backward walking mode," whereas the two rostral ganglia are in a "forward walking mode." These results augment previous observations by Bässler et al. (1985), who reported that hindlegs walk forward in the presence of front legs but backwards when the front legs have been amputated. This difference in metathoracic activity as a function of middle and front leg presence suggests that metathoracic segment walking direction is not determined by descending signals from the brain alone but also depends on the prothoracic and mesothoracic segments generating either stepping of the leg or forces through activation of leg muscles (Bässler et al., 1985). This difference in preferred direction during one-legged walking also suggests that the metathoracic ganglion walking CRG differs from the prothoracic and mesothoracic ones, at least under one-legged conditions, a finding that in general must be kept in mind when studying isolated parts of a nervous system (cf. Büschges et al., 1995).

\section{Segment specific reversal of the CS effect depends on walking direction}

Considerable knowledge has been gathered on the neural mechanisms underlying generation and control of rhythmic locomotor movements (Orlovsky et al., 1999; Pearson, 2000; Grillner, 2003) and how joint movement is coordinated in multisegmented locomotor organs (Hill et al., 2003; Büschges, 2005). However, up to now, this work has primarily involved studying how invariant, stereotyped movements are produced rather than how these movements are adapted in task-specific manners, even adaptations as simple as producing forward and backward walk- 
ing (Ayers and Davis, 1977; Graham and Epstein, 1985). We show here that the effect of leg loading reverses when walking direction changes from forward to backward. However, in each situation, the functional effect is to assist and promote stance phase motor output. Thus, these data show that the effects of the sensory inputs are switched in a context (forward vs backward walking) specific manner so as to maintain the same functional action (promoting stance). It is quite conceivable that the mechanism of context-dependent reflex reversal presented here can also be expanded to contribute to the turning behavior in insects. It has been shown previously in stick insects (Dürr and Ebeling, 2005) and cockroaches ( $\mathrm{Mu}$ and Ritzmann, 2005) that joint kinematics change from extension during stance to extension during swing in the inside leg during turning, whereas the outside leg remains the same as in forward walking. Given a unilateral organization of the leg muscle control systems in the stick insect, it is conceivable that the mechanisms for the reflex reversal, demonstrated here, could also contribute unilaterally in case of the turning.

Several neural mechanisms could underlie the reversal of the influence of load signals on coxal motoneuron activity. (1) Afferent fibers from load sensors could be affected by presynaptic inhibition (Stein and Schmitz, 1999), a mechanism that is present in a variety of locomotor systems that can drastically modify the gain of central sensory synapses (for review, see Clarac and Cattaert, 1996; Nusbaum et al., 1997). In the case at hand, presynaptic inhibition mediated by descending pathways from the front and middle legs and the brain could modify the gain of specific connections between load-sensing sensory neurons and ThC-joint CRG neurons in a phase-dependent manner and thereby switch the influence of CS signals on the ThC network. (2) Parallel pathways from CS to premotor interneurons could be shifted in weight and thereby reverse the influence of CS signals. In a number of motor systems, a readjustment of weight between antagonistic, parallel pathways from sense organs onto premotor interneurons and motoneurons has been shown to modify sensorimotor processing, including mediating reflex reversals (Büschges and El Manira, 1998; Clarac et al., 2000). Subsequent investigations are required to distinguish between these possibilities or explore others. Further work is also needed to determine whether, similar to the case with leg load sensors, the effects
A (i) forward walking

(ii) backward walking
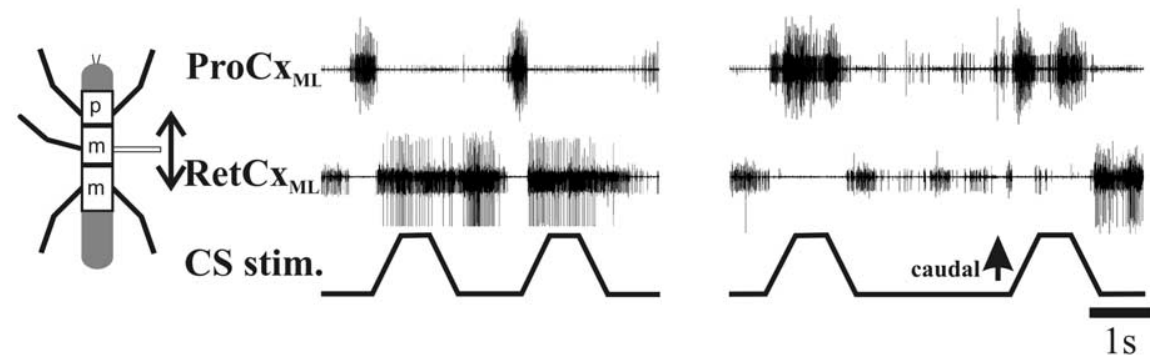

B

(i)

increased loading

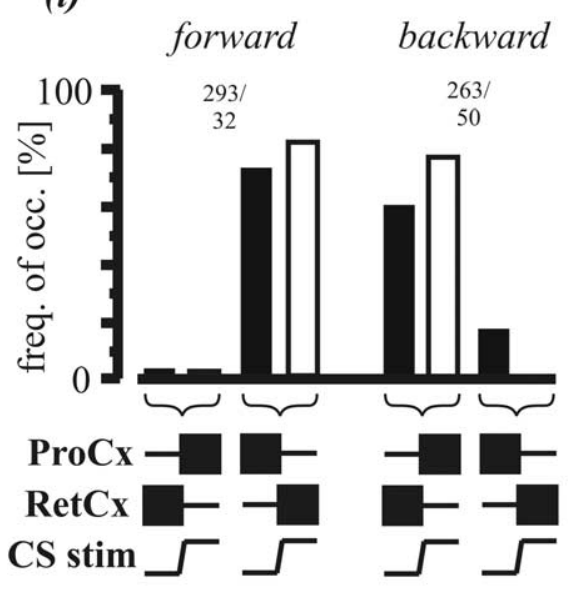

(ii)

released loading

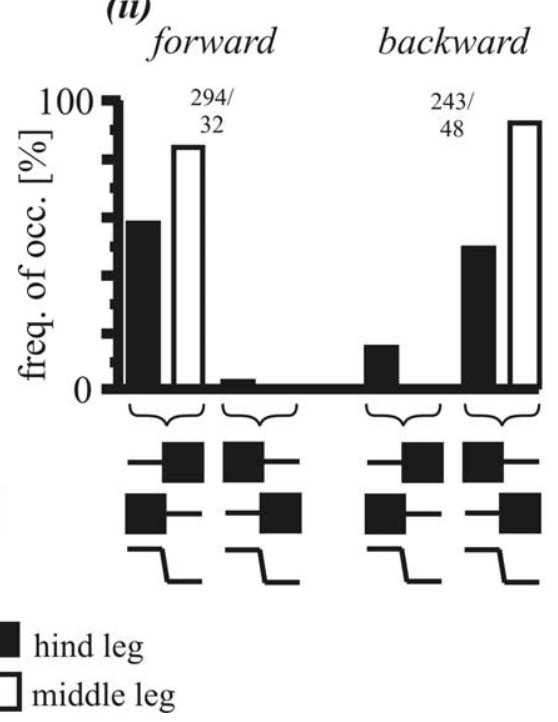

Figure 6. Influence of stimulation of middle and hindleg load sensors on coxal motoneuron activity as a function of walking direction. $\boldsymbol{A}$, Recording of RetCx and ProCx activity from the appropriate leg nerves of the mesothoracic segment with ongoing stimulation of load sensors. Note the reversed effect during forward (i) and backward (ii) walking. $\boldsymbol{B}$, Bar diagrams showing the occurrence frequency of transitions in motoneuron activity of the thoraco-coxal motoneurons in middle legs (white bars) and hindlegs (black bars) during stimulation of load sensors during forward and backward walking. $\boldsymbol{i}$, Increase in load; $\boldsymbol{i}$, decrease in load. Numbers above the columns indicate the number $(n)$ of stimuli evaluated for the hindleg (filled bars) and the middle leg (open bars), respectively. The sketches beneath the diagrams outline the two different cases, i.e., ProCx or Ret $C x$ motoneurons being currently active (bars) during caudal (upward deflection of the CS stim. trace) or rostral (downward deflection of the CS stim. trace) bending and the subsequent transition in activity.

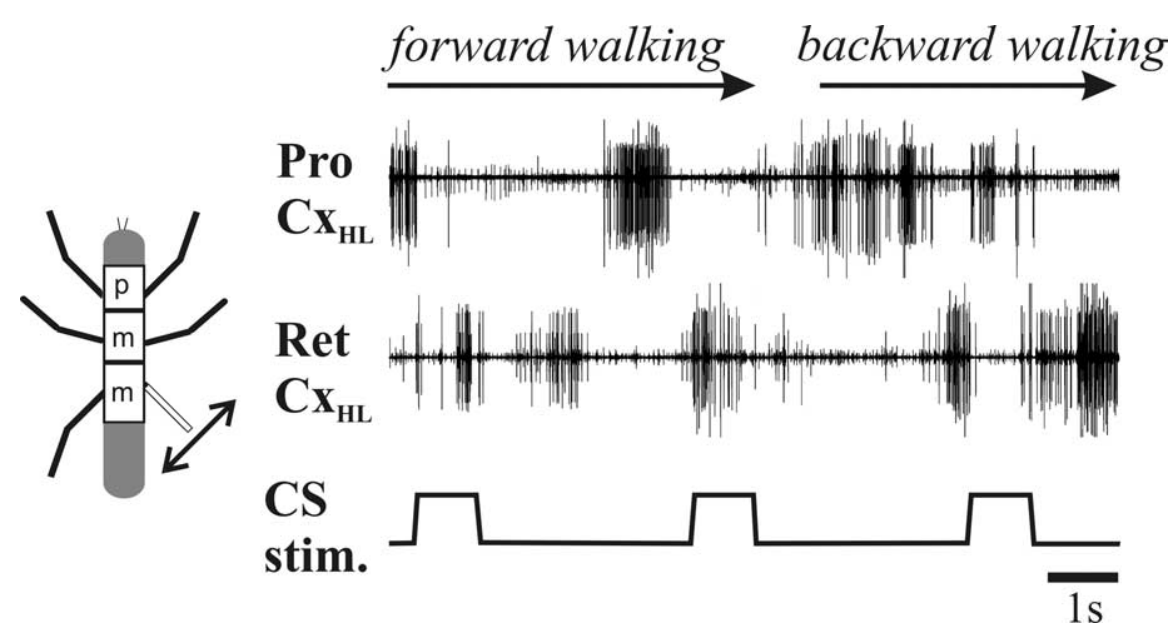

Figure 7. Extracellular recordings from hindleg ProCx and RetCx and CS stimulation during a section of a walking sequence showing that the reversal of the $C S$ effect occurs immediately when walking direction changes. 

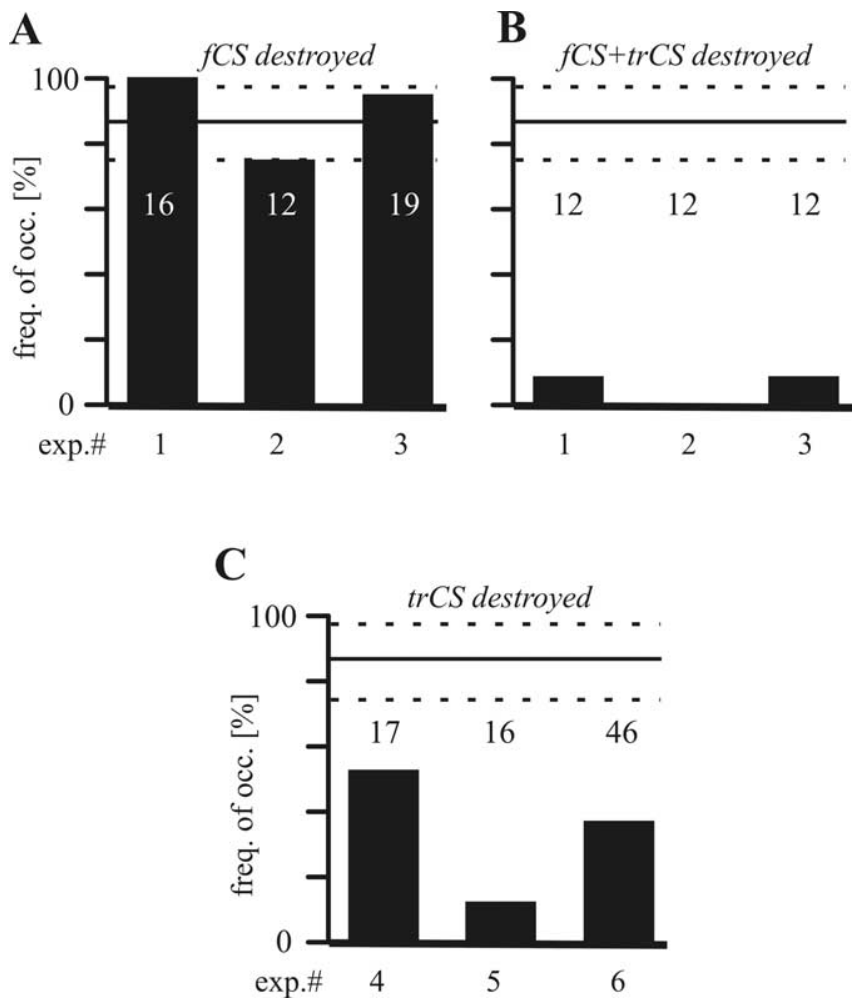

Figure 8. Effect of successive ablation of $\mathrm{fCS}$ and trCS on ThC-joint motoneuron activity in pilocarpine-induced rhythmic preparations in the middle leg ( $N=3$; the numbers on top of the bars give the sample size). In three animals (exp.\# 1-3), the influence of the bending of the femur persisted after removal of the fCS $(A)$. In these same animals, the reflex vanished after additional removal of the $\operatorname{tr} C(\boldsymbol{B}) . \operatorname{In} \boldsymbol{A}$ and $\boldsymbol{B}$, the vertical solid and dotted lines indicate the mean \pm SD from seven control experiments in which all CS were intact. The abscissa indicates the frequency of occurrence for transitions in switching from ProC $\mathrm{x}$ to RetCx activity induced by the CS stimulation. C, Effect of ablation of trCS only on ThC-joint motoneuron activity in pilocarpine-induced rhythmic preparations in the middle leg $(N=3$ ). In all three animals (exp.\# $4-6)$, the influence of the bending of the femur was strongly decreased after removal of the trCS.

of leg movement sensors are also reversed when walking direction switches. An intriguing possibility is that this reversal of sensory feedback is itself a principle and sufficient mechanism for generating forward and backward walking.

\section{References}

Akay T, Bässler U, Gerharz P, Büschges A (2001) Interjoint coordination: the role of sensory signals from the coxa-trochanteral joint on controlling motoneuronal activity of the femur-tibia joint in stick insects. J Neurophysiol 85:594-604.

Akay T, Haehn S, Schmitz J, Büschges A (2004) Signals from load sensors underlie interjoint coordination during stepping movements of the stick insect legs. J Neurophysiol 92:42-51.

Angel M, Guertin P, Jiménez I, McCrea DA (1996) Group I extensor afferents evoke disynaptic EPSPs in cat hindlimb extensor motoneurons during fictive locomotion. J Physiol (Lond) 494:851-861.

Ayers JL, Davis WJ (1977) Neuronal control of locomotion in the lobster Homarus americanus. J Comp Physiol 115:29-46.

Bartling CH, Schmitz J (2000) Reaction to disturbances of a walking leg during stance. J Exp Biol 203:1211-1233.

Bässler U (1977) Sense organs in the femur of the stick insect and their relevance to the control of position of the femur-tibia-joint. J Comp Physiol 121:99-113.

Bässler U (1986) Afferent control of walking movements in the stick insect
Cuniculina impigra. II. Reflex reversal and the release of swing phase in the restrained foreleg. J Comp Physiol A Neuroethol Sens Neural Behav Physiol 158:351-362.

Bässler U (1988) Functional principles of pattern generation for walking movements of stick insect forelegs: the role of the femoral chordotonal organ afferences. J Exp Biol 136:125-147.

Bässler U (1993) The femur-tibia control system of stick insects-a model system for the study of the neural basis of joint control. Brain Res Rev 18:207-226.

Bässler U, Büschges A (1998) Pattern generation for walking movements of stick insects-multisensory control of a locomotor program. Brain Res Rev 27:65-88.

Bässler U, Foth E, Breutel G (1985) The inherent walking direction differs for the prothoracic and metathoracic legs of stick insects. J Exp Biol 116:301-311.

Büschges A (2005) Sensory control and organization of neural networks mediating coordination of multisegmental organs for locomotion. J Neurophysiol 93:1127-1135.

Büschges A, El Manira A (1998) Sensory pathways and their modulation in the control of locomotion. Curr Opin Neurobiol 8:733-739.

Büschges A, Schmitz J, Bässler U (1995) Rhythmic patterns in the thoracic nerve cord of the stick insect induced by pilocarpine. J Exp Biol 198:435-456.

Cattaert D, LeRay D (2001) Adaptive motor control in crayfish. Prog Neurobiol 63:199-240.

Clarac F, Cattaert D (1996) Invertebrate presynaptic inhibition and motor control. Exp Brain Res 112:163-180.

Clarac F, Cattaert D, LeRay D (2000) Central control components of a "simple" stretch reflex. Trends Neurosci 23:199-208.

Cruse H (1985) Which parameters control leg movements of a walking leg? II. The start of swing phase. J Exp Biol 116:357-362.

Delcomyn F (1991) Activity and directional sensitivity of leg campaniform sensilla in a stick insect. J Comp Physiol A Neuroethol Sens Neural Behav Physiol 168:113-119.

DiCaprio RA, Clarac F (1981) Reversal of a walking leg reflex elicited by a muscle receptor. J Exp Biol 90:197-203.

Dürr V, Ebeling W (2005) The behavioural transition from straight to curve walking: kinetics of leg movement parameters and the initiation of turning. J Exp Biol 208:2237-2252.

Duysens J, Clarac F, Cruse H (2000) Load-regulating mechanisms in gait and posture: comparative aspects. Physiol Rev 80:83-133.

Ekeberg O, Blümel M, Büschges A (2004) Dynamic simulation of insect walking. Arthropod Struct Dev 33:287-300.

Epstein S, Graham D (1983) Behav and motor output for an insect walking on a slippery surface. 1. Forward walking. J Exp Biol 105:215-229.

Graham D (1985) Pattern and control of walking insects. Adv Insect Physiol 18:31-140.

Graham D, Bässler U (1981) Effects of afference sign reversal on motor activity in walking stick insects (Carausius morosus). J Exp Biol 91:179-193.

Graham D, Epstein S (1985) Behav and motor output for an insect walking on a slippery surface. 2. Backward walking. J Exp Biol 118:287-296.

Grillner S (2003) The motor infrastructure: from ion channels to neuronal networks. Nat Rev Neurosci 4:573-586.

Gruhn M, Hoffmann O, Dübbert M, Scharstein H, Büschges A (2006) Tethered stick insect walking: a modified slippery surface setup with optomotor stimulation and electrical monitoring of tarsal contact. J Neurosci Methods 158:195-206.

Hiebert GW, Whelan PJ, Prochazka A, Pearson KG (1996) Contribution of hind limb flexor muscle afferents to the timing of phase transitions in the cat step cycle. J Neurophysiol 75:1126-1137.

Hill AA, Masimo MA, Calbrese RL (2003) Intersegmental coordination of rhythmic motor patterns. J Neurophysiol 90:531-538.

Hofmann T, Bässler U (1982) Anatomy and physiology of trochanteral campaniform sensilla in the stick insect, Cuniculina impigra. Physiol Entomol 7:413-426.

Hofmann T, Bässler U (1986) Response characteristics of single trochanteral campaniform sensilla in the stick insect, Cuniculina impigra. Physiol Entomol 11:17-21.

Marquardt F (1940) Beiträge zur Anatomie der Muskulatur und der peripheren Nerven von Carausius (Dixippus) morosus. Zool JB Abt Anat Ont 66:63-128. 
Mu L, Ritzmann RE (2005) Kinematics and motor activity during tethered walking and turning in the cockroach, Blaberus discoidalis. J Comp Physiol A Neuroethol Sens Neural Behav Physiol 191:1037-1054.

Nusbaum MP, El Manira A, Gossard J-P, Rossignol S (1997) Presynaptic mechanisms during rhythmic activity in vertebrates and invertebrates. In: Neurons, Networks, and Motor Behavior (Stein PSG, Selverston AI, Stuart DG, eds) pp 237-253. Cambridge, MA: MIT.

Orlovsky GN, Deliagina TG, Grillner S (1999) Neural control of locomotion. From mollusk to man. Oxford, UK: Oxford UP.

Pearson KG (2000) Plasticity of neuronal networks in the spinal cord: modifications in response to altered sensory input. Prog Brain Res 128:61-70.

Pearson KG, Collins DF (1993) Reversal of the influence of group Ib afferents from plantaris on activity in medial gastrocnemius muscle during locomotor activity. J Neurophysiol 70:1009-1017.

Pringle JWS (1938) Proprioception in insects II. The action of the campaniform sensilla on the legs. J Exp Biol 15:114-131.

Schmitz J (1993) Load-compensating reactions in the proximal leg joints of stick insects during standing and walking. J Exp Biol 183:15-33.
Schmitz J, Haßfeld G (1989) The treading-on-tarsus reflex in stick insects: phase-dependence and modifications of the motor output during walking. J Exp Biol 143:373-388.

Schmitz J, Stein W (2000) Convergence of load and movement information onto leg motoneurons in insects. J Neurobiol 42:424-436.

Schmitz J, Büschges A, Delcomyn F (1988) An improved electrode design for en passant recordings from small nerves. Comp Biochem Physiol 91A:769-772.

Stein W, Schmitz J (1999) Multimodal convergence of presynaptic afferent inhibition in insect proprioceptors. J Neurophysiol 82:512-514.

Whelan PJ, Hiebert GW, Pearson KG (1995) Plasticity of the extensor group I pathway controlling the stance to swing transition in the cat. J Neurophysiol 74:2782-2787.

Weidler DJ, Diecke FPJ (1969) The role of cations in conduction in the central nervous system of the herbivorous insect Carausius morosus. $\mathrm{Z}$ Verl Physiol 64:372-399.

Zill SN, Schmitz J, Büschges A (2004) Load sensing and control of posture and locomotion. Arthropod Struct Dev 33:273-286. 